\title{
EOPs: Uso de la Proloterapia en pacientes con sindromes asociados a disfunción tendinosa crónica
}

\author{
Use of Prolotherapy in the treatment of chronic tendinopathy associated syndromes
}

Sergio Terrasa*, María del Valle Agüero** y Ricardo Frusso ${ }^{\star \star \star}$

\begin{abstract}
Resumen
La proloterapia es una técnica basada en la infiltración reiterada de sustancias irritantes en tendones y ligamentos deteriorados con el objetivo de desencadenar una respuesta inflamatoria que llevaría al fortalecimiento y regeneración de estas estructuras, reduciendo el dolor y recuperando su funcionalidad.

A partir de la consulta de una paciente con antecedentes de omalgia crónica sin respuesta satisfactoria a los tratamientos habituales, un médico de familia se plantea la posibilidad de realizar proloterapia como opción terapéutica. Se plantea entonces una pregunta sobre su efectividad y se realiza una búsqueda bibliográfica para encontrar la mejor evidencia disponible. Se describen cuatro publicaciones sobre el tratamiento de síndromes asociados a disfunción tendinosa. Si bien la evidencia encontrada es débil y no esta relacionada con la región afectada en la paciente en cuestión, representa la mejor con la que contamos para la toma de decisiones, sumada al buen juicio clínico, y a los valores de la paciente.

\section{Abstract}

Prolotherapy is a technique involving the repeated injection of irritants agents into ligaments and tendinous attachments, in order to trigger an inflammatory response that theoretically will lead to a subsequent strengthening of ligaments and a decrease in pain and disability. From the case of a patient presenting with chronic shoulder pain that was not relieved with standard treatments, a family physician considers a treatment strategy involving prolotherapy. Then a clinical question about its efficacy is posed and a literature search strategy is undertaken. It is summarized the evidence from four publications wich describe the efficacy of this treatment on chronic "tendinopathy associated syndromes". Although the results showed weak evidence and the procedures were not related with the aforementioned patient pain location, these represents the best evidence for the decision making process taking into account the patient values and a good clinical judgment.
\end{abstract}

Palabras clave: tendinopatía, tendinosis, proloterapia. Key words: tendinopathy, tendinosis, prolotherapy.

Terrasa S, Agüero M, Frusso R. Uso de la Proloterapia en pacientes con síndromes asociados a disfunción tendinosa crónica. Evid Act Pract Ambul. 13(2).7477. Abr-Jun 2010.

\section{Caso clínico}

Un médico de familia atiende a una mujer de 78 años con antecedentes de omalgia izquierda crónica y limitación de su funcionalidad. Usa a demanda y por la vía oral anti inflamatorios no esteroides (AINE), paracetamol, y $75 \mathrm{mg}$ de pregabalina en forma crónica. También ha realizado terapia física, sin resultados satisfactorios. Al examen físico se objetiva limitación a la movilidad activa de su hombro contra resistencia en las maniobras de evaluación de la fuerza muscular de los músculos supraespinoso y subescapular. Tiene una ecografía que informa que el tendón del músculo supraespinoso tiene una estructura difusamente heterogénea, con signos de ruptura parcial y que compromete el $30 \%$ de las fibras; que el del subescapular tiene estructura difusamente heterogénea y con áreas hipoecogénicas, compatible con tendinopatía crónica; y que el del infraespinoso tiene estructura difusamente heterogénea, compatible con tendinopatía leve. El médico le ofrece como opción de tratamiento la posibilidad de realizar proloterapia, que consiste en la infiltración con solución glucosada hipertónica al 25\% más lidocaína al 0,5\% en la articulación glenohumeral; y una solución glucosada hipertónica al 12,5\% con anestesia local a la misma dilución en la inserción temdinosa.

\section{Pregunta que generó el caso:}

En los pacientes con tendinopatía crónica (población), ¿la proloterapia (intervención) produce mejoría clínica con respecto a disminución del dolor y la discapacidad (resultado) comparándola con otras opciones terapéuticas y/o placebo (comparación)?

\section{Justificación de este artículo}

Los procesos musculoesqueléticos son la causa más frecuente de dolor, tanto agudo como crónico y de discapacidad temporal o permanente, para los que actualmente no hay un tratamiento universalmente efectivo. Vale destacar que, al menos un $30 \%$ de la población presenta algún síntoma en el aparato locomotor en el que el dolor de origen musculotendinoso tiene una importancia significativa ${ }^{1}$, estando la gravedad y la prevalencia de cada trastorno asociada con la edad.

Debido a que la población del mundo está envejeciendo, la búsqueda de nuevas terapias eficaces para estas enfermedades puede tener gran impacto en la atención del paciente individual y a nivel de la salud pública. Entre las opciones terapéuticas que suelen ofrecerse a los pacientes con dolores crónicos, se encuentra la proloterapia, clasificada actualmente dentro del grupo de las "Medicinas Complementarias y/o Alternativas", y según los expertos que la practican, reservada para pacientes con tendinopatías crónicas que no han mejorado con otros tratamientos; o bien en algunos pacientes con osteoartritis que no son candidatos a cirugía. A propósito de esta viñeta clínica y con el objetivo de contar con la mejor evidencia disponible sobre la efectividad de esta tecnología, hemos decidido hacer esta revisión bibliográfica para ayudar a los profesionales de la salud a compartir las decisiones terapéuticas con sus pacientes, lo que incluye poder aconsejarlos y permitirles elegir con autonomía.

\section{Estrategia de búsqueda}

Fueron realizadas en PubMed cinco búsquedas bibliográficas

* Servicio de Medicina Familiar y Comunitaria del Hospital Italiano de Buenos Aires. Departamento de Salud Pública del Instituto Universitario Hospital Italiano. sergio.terrasa@ hosptitalitaliano.org.ar

** Residencia de Medicina Familiar y General. Centro de Adiestramiento Dr. René Favaloro, departamento Rawson, San Juan.

${ }_{* \star *}$ Servicio de Medicina Familiar y Comunitaria del Hospital Italiano de Buenos Aires. 
con diferentes combinaciones de las siguientes palabras claves incluidas en el tesauro (MESH): "Shoulder", "Shoulder Pain", "Tendinopathy", "Tendinopathy/therapy", "Injections", "Injections, Intra-Articular"; y con la palabra "prolotherapy" en texto libre, ya que no figura en el tesauro. Se comenzó con estrategias restringidas, que inicialmente no arrojaron resultados hasta que, ampliando el espectro se obtuvieron un total de 23 citas, la mayoría correspondientes a trabajos que habían evaluado tratamientos con infiltraciones con corticoides. No se hallaron trabajos que hubieran evaluado la eficacia de la proloterapia en síndromes dolorosos del hombro. Se decidió entonces dirigir la búsqueda hacia el tratamiento de las tendinopatías en general. Se buscó en PUBMED empleando el filtro metodológico para artículos de revisión sistemática y empleando como palabras clave "prolotherapy", "injection therapy", "chronic musculoskeletal pain", "tendinopathy" y con las mismas palabras en TRIPDATABASE y la Biblioteca COCHRANE en Inglés. Esta búsqueda arrojó dos principales hallazgos: una revisión sistemática ${ }^{2}$ sobre la evidencia de la efectividad de la proloterapia para el tratamiento del dolor lumbar y otra referida a los problemas músculoesqueléticos en general ${ }^{3}$, así como una revisión narrativa ${ }^{4}$ sobre la proloterapia en Atención Primaria hecha por el autor de esta última ${ }^{3}$ y que se basaba en los trabajos encontrados en su revisión sistemática. Sin lograr hallar otros trabajos originales, los autores de este EOP revisaron las cuatro publicaciones que habían sido revisadas por aquel autor, que serán resumidas a continuación luego de una sucinta descripción de la tecnología.

\section{Descripción de la tecnología: Proloterapia}

La proloterapia (cuyo nombre proviene del concepto de "terapia de proliferación") es un tratamiento para el dolor musculoesquelético crónico basado en infiltraciones. Se supone que mejora la estabilidad articular mediante el fortalecimiento de los tendones o ligamentos estirados o desgarrados ${ }^{5}$ y consiste en la inyección de una solución irritante (que induce la proliferación) en los ligamentos y/o en las inserciones tendinosas con intervalos semanales, quincenales o mensuales (en general con un total de tres a ocho aplicaciones).

Los partidarios de la proloterapia ${ }^{6}$ consideran que las inyecciones en los ligamentos desencadenan un influjo de granulocitos, macrófagos y fibroblastos, la liberación de factores de crecimiento, y en último término, el depósito de colágeno de mejor calidad que el de las estructuras tendinosas o ligamentarias enfermas. Formulan la hipótesis de que este proceso conduce al fortalecimiento de los ligamentos y a una reducción del dolor y la discapacidad. Si bien habría cierta superposición de sus acciones, se describen tres clases principales de proliferantes comúnmente utilizados en la proloterapia ${ }^{7}$ : los irritantes, los quimiotácticos y los osmóticos ${ }^{\dagger \dagger}$.

Los más comúnmente usados en nuestro medio son los osmóticos y en forma de soluciones glucosadas de diferente concentración. A menudo, se agrega un anestésico local (comúnmente lidocaína) para reducir el dolor de las inyecciones irritantes y para poder constatar luego del procedimiento, que la solución terapéutica haya sido inyectada en el lugar correcto, lo que debería traducirse en que a los pocos minutos después de la infiltración y durante el período de duración de la acción del anestésico local (unas horas) el paciente sienta menos dolor. Durante la primera semana posterior a la infiltración es frecuente documentar que el paciente empeora su dolor, estando firmemente desaconsejado el uso de anti-inflamatorios no esteroides o aspirina -durante todo el tratamiento, ya que tenderían a suprimir o a atenuar el efecto inflamatorio del tratamiento local instituido y por lo tanto, a disminuir su eficacia en el largo plazo. La mayoría de los expertos recomienda el uso de paracetamol, o eventualmente opiáceos u otros tratamientos moduladores del dolor.

No hemos encontrado reportes respecto del riesgo de infección local asociado a la proloterapia intra-tendionosa. La mayoría de los expertos sostienen que sería mínimo en manos de un operador entrenado y utlizando una técnica aséptica. Para orientarnos, podríamos tomar como referencia la probabilidad de artritis séptica asociada a la inyección intra-articular de corticoides que está estimada en 1/10000 procedimientos $^{8}$, si bien la hiperosmolaridad de las soluciones habitualmente utilizadas en proloterapia haría suponer que este riesgo sería menor aún.

\section{Evidencia que sustenta el uso de la proloterapia}

La tabla 1 resume la evidencia que los autores de este artículo pudieron identificar sobre la eficacia de la proloterapia en el tratamiento de síndromes asociados a disfunción tendinosa con proloterapia.

Referidos específicamente a síndromes probablemente asociados a disfunción tendinosa del hombro y como antecedente histórico, mencionamos dos reportes de pocos casos de Shuman ${ }^{9,10}$ "que habían sido incluidos por Rabago y col. en la revisión sistemática antes mencionada ${ }^{3}$.

\section{Discusión}

Respecto de los profesionales de la salud en su práctica individual, la Medicina Basada en la Evidencia intenta ayudarlos en la toma de decisiones frente a los problemas de sus pacientes basándose en tres pilares básicos: 1) el buen juicio, la destreza clínica y la experiencia del profesional y/o el equipo actuante; 2) la evidencia científica disponible que respalda las intervenciones en salud que aparecen como opciones para el paciente en cuestión; 3) los valores del paciente y/o su comunidad.

La evidencia actual sólo respalda con fuerza -a través de los resultados de un pequeño ensayo clínico controlado ${ }^{11}$ y aleatorizado- la efectividad de la proloterapia en el tratamiento de la tendinopatía de los extensores de la muñeca o tendinosis del epicóndilo o epicondilosis lateral o "codo de tenista". Por otro lado y como vimos en la tabla 1, tres series de casos sugieren que este tratamiento podría ser beneficioso para otros síndromes asociados a disfunción tendinosa (la tendinopatia Aquileana ${ }^{12}$, la de los aductores de la cadera ${ }^{13} \mathrm{y}$ la fascitis plantar $\left.{ }^{14}\right)$.

Quienes propugnamos que las decisiones en salud sean tomadas basadas en la evidencia, estamos acostumbrados a leer artículos de más peso metodológico (ej. grandes ensayos clínicos aleatorizados y controlados contra placebo para el

\footnotetext{
${ }^{\dagger \dagger}$ Los irritantes (fenol, guayacol y ácido tánico) provocan directamente el daño de las células o el cambio a células antigénicas mediante la alteración de las proteínas superficiales. Los quimiotácticos (el único es el morruato de sodio) directamente atraen células inflamatorias. La clase osmótica de proliferantes incluye soluciones concentradas de glucosa, glicerina y sulfato de cinc; que provocan que las células liberen sustancias proinflamatorias.

$\pi$ Uno data de 1941 e incluyó el reporte exitoso de cuatro casos a de luxación recurrente tratados con inyecciones capsulares de Linsoleato de Sodio, Alparene, Silnasol y/o Neoplasmodio, que mejoraron su dolor y no volvieron a tener recurrencia de su luxación. El otro es de 1954 e incluyó 93 adultos con dolor lumbar bajo, gonalgia y "separación del hombro"; y reportó que el $95 \%$ de los pacientes pudo volver a trabajar.
} 
tratamiento de la hipertensión, el colesterol, etc.) por lo que la evidencia proveniente de los trabajos que acabamos de describir aparece como muy débil.

Sin embargo, a la hora de tomar decisiones para aliviar los síntomas o mejorar la funcionalidad o la calidad de vida de nuestros pacientes, uno debe tomarlas con la mejor evidencia bibliográfica disponible. En este caso, se trata de una paciente con un síndrome de dolor e impotencia funcional asociado a una disfunción tendinosa de la articulación del hombro, ya que la semiología del dolor coincide con la documentación ecográfica de la tendinopatía del músculo supraespinoso y del subescapular. Por otro lado, si bien no existen trabajos que hayan documen- tado la eficacia de la proloterapia en el tratamiento del los síndromes asociados a tendinopatías del hombro, sí la existe para otros síndromes que podrían analo-garse en tres aspectos a saber: 1) en su probable fisiopatogenia a través de una degeneración tendinosa asociada a la formación de fibras colágenas de peor calidad; 2) en su semio-logía (dolor al estresar la unidad músculo-tendinosa que se manifiesta con un empeoramiento del dolor cuando se dice al paciente que contraiga el músculo contra resistencia y/o a la compresión de la inserción tendinosa en el hueso); 3) en la documentación ecográfica de una estructura tendinosa con degeneración de sus fibras que corresponde con la semiología previamente descripta.

Tabla 1: descripción de la calidad de la evidencia que sustenta el uso de proloterapia en diferentes síndromes clínicos asociados a tendinopatías.

\begin{tabular}{|c|c|c|c|c|}
\hline $\begin{array}{l}\text { Tendinopatía evaluada, } \\
\text { autor, diseño y "n" }\end{array}$ & $\begin{array}{l}\text { Criterios de } \\
\text { inclusión }\end{array}$ & $\begin{array}{l}\text { Intervención (I) y } \\
\text { comparación (C) }\end{array}$ & $\begin{array}{l}\text { Nro de inyecciones, periodicidad, lugar } \\
\text { de administración y evaluación }\end{array}$ & Resultados \\
\hline $\begin{array}{l}\text { De los extensores de la } \\
\text { muñeca (epicondilosis } \\
\text { lateral o "codo de } \\
\text { tenista"). } \\
\text { Scarpone y col.' } \\
\text { ECCA } \\
20 \text { adultos }\end{array}$ & $\begin{array}{l}\text { Epicondilosis o dolor de } \\
\text { codo de más de seis } \\
\text { meses de evolución, } \\
\text { refractario al reposo, } \\
\text { AINE, infiltraciónes con } \\
\text { corticoides y terapia } \\
\text { física. }\end{array}$ & $\begin{array}{l}\text { I: Dextrosa al } 10,7 \%, \\
\text { morruato de sodio al } \\
14,7 \% \text { y lidocaína } 4 \% \\
\text { C: solución fisiológica }\end{array}$ & $\begin{array}{l}\text { Tres inyecciónes en la cresta } \\
\text { supracondílea, en el ligamento del } \\
\text { epicóndilo lateral y en el ligamento anular a } \\
\text { las } 0,8 \text { y } 16 \text { semanas y evaluación a las } 52 \\
\text { semanas con seguimiento telefónico con } \\
\text { tres preguntas. }\end{array}$ & $\begin{array}{l}\text { Primario: mejoría significativa del puntaje de dolor } \\
\text { en el grupo proloterapia }(5,1 \pm 0,8,3,3 \pm 0,9 \text { y } \\
0,5 \pm 0,4) \text { versus }(4,5 \pm 1,7,3,6 \pm 1,2 \text { y } 3,5 \pm 1,5) \text { en el } \\
\text { grupo placebo (basal, } 8 \text { y } 16 \text { semanas } \\
\text { respectivamente). Disminución de } 3,6 \text { puntos en la } \\
\text { EAV } 0-10 \text {, con un NNT de } 1,4 \text { para disminuir dos } \\
\text { puntos en la escala de dolor. } \\
\text { Secundario: mejoría en la fuerza isométrica sin } \\
\text { diferencias estadísticamente significativas entre } \\
\text { ambos grupos. }\end{array}$ \\
\hline $\begin{array}{l}\text { Aquileana } \\
\text { Maxwell y col. } \\
\text { Serie de casos } \\
36 \text { adultos }\end{array}$ & $\begin{array}{l}\text { Tendinosis de mas de tres } \\
\text { meses) con fracaso en } \\
\text { múltiples tratamientos } \\
\text { (ej. fisioterapia e } \\
\text { inyección con esteroides). }\end{array}$ & $\begin{array}{c}\text { I: Dextrosa al } 25 \% \text { y } \\
\text { lidocaína } 2 \%\end{array}$ & $\begin{array}{l}\text { Inyecciones intra-tendinosas bajo control } \\
\text { ecográfico en áreas anormales hipoecoicas } \\
\text { y hendiduras anecoicas o en la porción } \\
\text { engrosada del tendón, cada seis semanas, } \\
\text { finalizando cuando resolvia el dolor o si } \\
\text { luego de cuatro inyección. }\end{array}$ & $\begin{array}{l}\text { Primario: reducción estadísticamente significativa } \\
\text { del porcentaje del dolor medido con tres EAV 0-100. } \\
\text { EAV1: } 88,2 \% \text {; EAV2: 84,0\%; EAV3: 78,1\%. Para } \\
\text { todos ( } p<0,0001) \text {. } \\
\text { Secundario: el espesor ecográfico del tendón } \\
\text { disminuyó de un promedio de 11,7 a 11,1 mm ( } p \\
<0,007) \text {. El nro de tendones con hendiduras } \\
\text { anecoicas o focos se redujo en un } 78 \% \text {; y la } \\
\text { neovascularización disminuyó en } 18 \text { tendones } \\
\text { (55\%). }\end{array}$ \\
\hline $\begin{array}{l}\text { Aductores de la cadera } \\
\text { Topol y col.' } \\
\text { Serie de } 24 \text { casos } \\
\text { ( } 22 \text { rugbiers y } 2 \\
\text { futbolistas) }\end{array}$ & $\begin{array}{l}\text { Dolor inguinal crónico } \\
\text { refractario a inyección } \\
\text { con esteroides y terapia } \\
\text { física. }\end{array}$ & $\begin{array}{l}\text { I: Dextrosa al 12,5\% y } \\
\text { lidocaína al 0,5\% } \\
\text { C: Ausente }\end{array}$ & $\begin{array}{l}\text { Una inyección al inicio y luego cada un mes } \\
\text { en los orígenes del muslo aductor } \\
\text { (inserciones abdominal y suprapúbica) y en } \\
\text { la sínfisis del pubis, hasta la remisión } \\
\text { completa del dolor o la falta de mejoría } \\
\text { luego de dos inyecciones. }\end{array}$ & $\begin{array}{l}\text { Primario: reducción estadísticamente significativa } \\
\text { del dolor. } \\
\text { EAV 0-10: de un basal } 6,3 \pm 1,4 \text { a } 1,0 \pm 2.4 \text { post } \\
\text { tratamiento; con una diferencia de }-5,3 \text { (IC95\% }-6,3 \\
\text { a -4,3) puntos. } \\
\text { NPPS: de un basal de } 5,3 \pm 0,7 \text { a } 0,8 \pm 1,9 \text { post } \\
\text { tratamiento; con una diferencia de }-4,5 \text { (IC95\% }-5,1 \\
\text { a -3,9) puntos. }\end{array}$ \\
\hline $\begin{array}{l}\text { Fascitis plantar } \\
\text { Ryan y col. } \\
\text { Serie de } 20 \text { casos }\end{array}$ & $\begin{array}{l}\text { Fascitis plantar crónica } \\
\text { (seis meses) refractaria al } \\
\text { tratamiento con AINE, } \\
\text { corticoides, fisioterapia y } \\
\text { otros. }\end{array}$ & $\begin{array}{l}\text { I: Dextrosa al } 25 \% \text { y } \\
\text { lidocaína al } 2 \%\end{array}$ & $\begin{array}{l}\text { Una a tres inyecciones intraligamentarias } \\
\text { bajo guía ecográfica en areas ligamentarias } \\
\text { anormalmente engrosadas y/o hipoecoicas } \\
\text { con intervalo de seis semanas y } \\
\text { seguimiento telefónico a los } 12 \text { meses. }\end{array}$ & $\begin{array}{l}\text { Primario: reducción estadísticamente significativa } \\
(\mathrm{p}<0.001) \text { del dolor en EAV 0-100. EAV1: } 36,8 \text { (DS } \\
25,6) \text { a } 10,3(10,9) \text {. } \\
\text { EAV2: } 74,7(20,8) \text { a } 25,0(27,7) \text {. EAV3: } 91,6(9,2) \text { a } \\
38,7(35,1) .\end{array}$ \\
\hline
\end{tabular}

ECCA: ensayo clínico controlado y aleatorizado. EAV 0-10: escala analógica visual de 0 a 10 puntos. EAV 0-100: escala visual analógica de 0 a 100 puntos. EAV1: dolor en reposo. EAV2: dolor durante la actividad diaria normal. EAV3: dolor durante o después del deporte u otra actividad física. DS: desvío Standard. NPPS: Escala de fases de dolor de Nirschl. NNT: numero necesario a tratar.

a Scarpone M y col. The efficacy of prolotherapy for lateral epicondylosis: a pilot study. Clinical J Sports Med. 2008;18:248-254.

${ }^{\mathrm{b}}$ Maxwell $\mathrm{N}$ y col. Sonographically guided intratendinous injection of hyperosmolar dextrose to treat chronic tendinosis of the Achilles tendon: a pilot study. Am J Roent. 2007;189(4):W215-220.

' Topol G y col. Efficacy of dextrose prolotherapy in elite male kicking-sport athletes with groin pain. Arch Phys Rehabil. 2005;86:697-702.

${ }^{d}$ Ryan M y col. Sonographically guided intratendinous injections of hyperosmolar dextrose/lidocaine: a pilot study for the treatment of chronic plantar fasciitis. Br J Sports Med. 2009;43:303-306. doi: 10.1136/bjsm.2008.050021 
Si bien la evidencia que hemos resumido aparece como débil, vale destacar que uno de los autores de este artículo ha tenido la experiencia de observar excelentes resultados con proloterapia en varios pacientes con omalgia crónica de presentación clínica similar a la que hemos descripto en la viñeta inicial, algunos de ellos tratados por el mismo, y otros, en el contexto de la observación clínica de dicha técnica en oportunidad de estar entrenándose en su aplicación con un experto en la técnica (otro de los autores). Para complicar más aún la situación, hay que aclarar que la eficacia de este tipo de tratamiento es altamente dependiente de la destreza del operador que lo administra. Los expertos en proloterapia sostienen que es importante aplicar con precisión el agente irritante en la zona tendinosa afectada que está siendo responsable de la manifestación clínica que se procura aliviar. Esto implica una doble habilidad: 1) un buen conocimiento semiológico de los síndromes músculotendinosos para establecer con buen nivel de certeza cuál es la estructura afectada que está implicada en el síntoma del paciente; 2) destreza en la aplicación del agente irritante para que este llegue al lugar donde debe ejercer su efecto.

¿Entonces, vale ofrecer tratamiento con proloterapia para un síndrome de disfunción tendinosa (de una región del cuerpo) si sólo existe documentación de su eficacia para otros síndromes de disfunción tendinosa (de otra región del cuerpo)?

No es fácil la respuesta. Volviendo a los conceptos que comenzamos a desarrollar al inicio de la discusión de este artículo, consideramos que frente al caso individual y si es que el equipo tratante tiene destreza en su instrumentación y experiencia de buenos resultados, es lícito hacerlo siempre que el paciente sea informado de la limitación de la Evidencia existente. Justificamos esta posición argumentando que en la práctica médica diaria, las decisiones se toman sobre la base de la mejor evidencia disponible, de la experiencia y el juicio clínico del equipo profesional y de los valores del paciente.

\section{Evolución del caso}

La paciente aceptó el tratamiento ofrecido. Sin abandonar el tratamiento kinesiológico y partiendo de un dolor que antes de la primera infiltración había sido calificado como severo, luego de dos inyecciones recibidas con intervalo de dos meses, el dolor y la funcionalidad mejoraron considerablemente y la satisfacción de la paciente fue alta.

\section{Conclusión de los autores}

Tanto la paciente como el equipo tratante nos hemos alegrado con el resultado de la estrategia ofrecida, que no difiere de otros casos que hemos tratado previamente. Sin embargo, esperamos con avidez los resultados de trabajos futuros que ayuden a echar luz sobre la efectividad de esta tecnología terapéutica cada vez más difundida y de la que contamos todavía con poca evidencia bibliográfica.

Recibido el 02/05/2010 y Aceptado el 23/06/2010

\section{Referencias}

1. Barutell Farinos C y col. Sindrome de dolor miofascial Grupo de Trabajo Multidisciplinar para la elaboración de una Guía Clínica para la Evaluación y Tratamiento del Dolor Miofascial 2007

2. Dagenais $S$ y col. Prolotherapy injections for chronic low-back pain. Cochrane Database Syst Rev. 2007 Apr 18;(2):CD004059. Review.PMID: 17443537.

3. Rabago D y col. A systematic review of prolotherapy for chronic musculoskeletal pain. Clin J Sports Med 2005, 15:376-380.

4. Rabago D y col. Prolotherapy in primary care practice. Department of Family Medicine, University of Wisconsin School of Medicine and Public Health,2010 Mar;37(1):65-80. 5. Klein R y col. Biochemical injection treatment for discogenic low back pain. The Spine Journal 2003; 3:220-6.

6. Rabago D y col. A systematic review of four injection therapies for lateral epicondylosis: prolotherapy, polidocanol, whole blood and platelet-rich plasma. Br J Sports Med. 2009 Jul;43(7):471-81. Epub 2008 Nov 21.PMID: 19028733.

7. Banks A. A rational for prolotherapy. Journal of Orthopaedic Medicine 1991;13(3):54-9.

8. Hunter J y col. A Risk-Benefit Assessment of Intra-Articular Corticosteroids in Rheumatic Disorders. Drug Safety. 21(5). Nov 1999, pp. 353-365(13).

9. Shuman D. Luxation recurring in the shoulder. Osteopath Prof. 1941;8: 11-14. Citada por: Rabago D y col. A systematic review of prolotherapy for chronic musculoskeletal pain. Clin J Sports Med 2005, 15:376-380.

10. Shuman D. Sclerotherapy: statistics on its effectiveness of unstable Joint conditions. Osteopath Prof. 1954;21:11-15, 37-38. Citada por: Rabago D y col. A systematic review of prolotherapy for chronic musculoskeletal pain. Clin J Sports Med 2005, 15:376-380.

11. Scarpone M y col. The efficacy of prolotherapy for lateral epicondylosis: a pilot study. Clinical J Sports Med. 2008;18:248-254.

12. Maxwell $\mathrm{N}$ y col. Sonographically guided intratendinous injection of hyperosmolar dextrose to treat chronic tendinosis of the Achilles tendon: a pilot study. Am $\mathrm{J}$ Roent. 2007;189(4):W215-220.

13. Topol G y col. Efficacy of dextrose prolotherapy in elite male kicking-sport athletes with groin pain. Arch Phys Rehabil. 2005;86:697-702.

14. Ryan $\mathrm{M}$ y col. Sonographically guided intratendinous injections of hyperosmolar dextrose/lidocaine: a pilot study for the treatment of chronic plantar fasciitis. Br $\mathrm{J}$ Sports Med. 2009;43:303-306. doi: 10.1136/bjsm.2008.050021 